Stoa

Vol. 3, no. 6, 2012, pp. 139-160

ISSN 2007-1868

\title{
AFECTIVIDAD Y COGNICIÓN. HACIA UNA NUEVA IDEA DE AGENTE EPISTÉMICO
}

\author{
Ana Luisa Ponce Miotti \\ Universidad Veracruzana \\ luanas@gmail.com
}

RESUMEN: En el ámbito de la epistemología, la manera de entender el vínculo entre afectividad y cognición ha sufrido transformaciones importantes a partir de las cuales lo central ya no es la racionalidad de la afectividad sino la afectividad de lo racional. Nuestra propuesta busca incorporarse a estos trabajo y tiene como objetivo esbozar la manera en que creemos se establece este vínculo entre afectividad y cognición.

Para esto, en un primer momento expondremos, de manera general, qué entendemos por afectos, estableciendo una cierta analogía entre afectividad y percepción. A partir de este primer desarrollo, consideraremos la manera en que la afectividad interactúa con otros estados cognitivos, en particular, con las creencias.

Este tratamiento otorgará, como resultado último, una visión particular de los procesos cognitivos, los cuales son ejercidos por un agente complejo y situado en una determinada práctica epistémica.

PALABRAS ClAVE: Afectividad · cognición · creencias · agente epistémico

ABSTRACT: In the field of epistemology, the way in which the link between emotion and cognition is understood has undergone major transformations from which, the focus is no longer the rationality of the emotions but the emotions of rationality. Our proposal seeks to incorporate these works and aims as well to outline how we understand this link between emotion and cognition.

For this, at first we will discuss what we mean by affects, setting a certain analogy between emotion and perception. This first development will allow us to consider how the emotions interact with other cognitive states, in particular beliefs. 
This treatment will give as result a particular view of cognitive processes, which is exercised by a complex agent and located in a particular epistemic practice.

KeYwORDS: Affectivity $\cdot$ cognition $\cdot$ belief $\cdot$ epistemic agent

\section{Presentación}

Si bien el tema de la afectividad posee profundas raíces en la tradición filosófica, la manera en que se ha tratado el problema no siempre significó una concepción positiva de los afectos, en particular en lo referente al vínculo entre los estados afectivos y la cognición. Ciertamente, el supuesto de que los afectos no tienen ningún papel en los procesos cognitivos, o incluso, de que son un obstáculo para éstos, es una visión muy arraigada en la historia de la filosofía. La razón y los afectos, según estas visiones, pertenecen a ámbitos distintos, cuyo vínculo, en caso de existir, se basa en una relación de subordinación y dominio. En esta lucha por el control se espera que la razón ejerza la autoridad ante un espíritu pasional que confunde y distorsiona la realidad dificultando toda posibilidad de conocimiento.

Si bien este tratamiento encuentra ciertas excepciones -como por ejemplo en la propuesta Aristotélica-, la concepción dominante es una idea de racionalidad desencarnada y desapasionada, a todas luces herencia de la modernidad, en particular dentro de los ámbitos que nos ocupan: la filosofía de la ciencia y la epistemología. Efectivamente, dejando de lado algunos antecedentes dentro de la filosofía de la ciencia de principios del siglo $\mathrm{xx}$, como por ejemplo los trabajos de Neurath (1913), Duhem (1914) y Polanyi (1958), esta rama de la filosofía se ha caracterizado por asimilar los afectos como disturbios de la razón, es decir, estos se asumen como estados que no sólo se oponen a la racionalidad sino que pueden ser un obstáculo para ésta.

Este entendimiento se encuentra más arraigado en el ámbito epistemológico, donde no fue sino hasta 1980 cuando se produjo un giro claro y decisivo respecto al entendimiento de los afectos y su papel en la cognición. No obstante, la manera en que se produjo dicho giro fue a través del análisis y la demostración del aspecto racional de la afectividad, es decir, se buscó su rescate manteniéndola dentro de los límites marcados por la tradición; de aquí que, para estos primeros intentos dentro de la epistemología, la única forma de transformar el estatus de 
los afectos es demostrando su racionalidad. Como afirma Elgin (1996), "estos intentos terminan asumiendo una concepción muy estrecha del ámbito cognitivo" (p. 146).

Sin embargo, en las últimas décadas este entendimiento ha sufrido transformaciones importantes, a partir de las que lo central ya no es la racionalidad de la afectividad, sino la afectividad de lo racional, tal es el caso de los trabajos de De Sousa (1987), Damasio (1994), Elster (1999), Pérez Ransaz (2011), entre otros.

Nuestra propuesta busca incorporarse a estos desarrollos últimos, siendo el objetivo esbozar la manera en que creemos se establece este vínculo entre afectividad y cognición. Con esto, citando a Elgin, "nuestro objetivo no será anestesiar la afectividad sino sensibilizar la cognición" (1996, p. 147). Para esto, en un primer momento expondremos, de manera general, qué entendemos por afectos, estableciendo una cierta analogía entre afectividad y percepción. A partir de este tratamiento consideraremos la manera en que la afectividad interactúa con otros estados cognitivos, en particular, con las creencias.

Este desarrollo otorgará como resultado una visión particular de los procesos cognitivos, ejercidos por un agente complejo y situado en una determinada práctica epistémica.

\section{La afectividad como percepción}

Partimos del supuesto de que los afectos son estados que se asemejan a la percepción al determinar patrones de notoriedad, es decir, al hacer más prominentes ciertos rasgos de la experiencia que pasarían desapercibidos en ausencia de dichos estados afectivos. Para comprender este papel de la afectividad retomamos la noción de "escenarios afectivos" propuesta por De Sousa (1982), con ésta, alude a situaciones que van conformando nuestra afectividad al familiarizarnos por asociación con el vocabulario de los afectos desde la formación más temprana, posibilitada por condiciones biológicas innatas y reforzados, posteriormente, por las historias, el arte y la cultura en general, a la cual estemos expuestos. De aquí que dichos escenarios sean considerados, por una parte, una respuesta biológica natural a la situación paradigmática, y por otra, una respuesta culturalmente adquirida.

$\mathrm{Al}$ respecto, como bien afirma Goldie (2000), para cada clase de experiencia afectiva habrá respuestas paradigmáticas envolviendo pen- 
samientos, motivaciones y sentimientos, así como cambios corporales, actividad expresiva, acciones, etc., y esto, será epistemológicamente central a la concepción de afecto y a cómo aprendemos a aplicarlo (p. 33). ${ }^{1}$ Para aclarar estas idea, una analogía pertinente es la percepción de cualidades tales como el color, el tono o el olor; el modo en que las aprehendemos a través de los sentidos no es fija ni uniforme (un agente puede percibir variaciones en alguna melodía que otro, quizás menos educado en la música, no percibe) con lo cual se asume que este tipo de percepción puede ser educada y refinada; sin embargo, a pesar de la variabilidad y falibilidad en la percepción de dichas cualidades, éstas proporcionan un acceso epistémico al objeto.

Esta manera de entender los afectos tiene diversas ventajas respecto a otras propuestas sobre la materia, en particular, respecto a ciertos desarrollos dentro de la línea cognitivista que asumen una concepción escolástica de la intencionalidad, por la que sostienen que los afectos poseen una dirección, y se orientan hacia un objeto particular. Acorde a estas posturas, el objeto intencional, el cual es independiente del estado afectivo en cuestión, funge como el elemento que provoca y se le dirige un determinado estado afectivo. Siguiendo a Solomon (2004) esta noción tradicional de intencionalidad no hace justicia a la característica de compromiso con el mundo que distingue a los afectos: estos "no son sólo sobre (o dirigidos a) el mundo, sino que están activamente ‘engranados' en él”. (p. 77).

$\mathrm{Al}$ respecto, asumimos que el objeto formal del afecto es una cualidad de segundo orden, cuyo origen debe explicarse a partir de los elementos que conforman un escenario afectivo; así, la intencionalidad afectiva, desde nuestra concepción, involucra cualidades tales como "horroroso", "odioso", "amoroso", etc., lo que permite, a diferencia de las aludidas propuestas que refieren a una intencionalidad dirigida a un objeto o situación concreta e independiente del estado afectivo, incluir un espectro más amplio cuando nos referimos a la afectividad; es decir, teniendo en cuenta la clasificación prevaleciente dentro de las propuestas filosóficas, estamos incluyendo tanto las emociones, como

\footnotetext{
${ }^{1}$ En este punto es necesario aclarar que tanto Sosa como Goldie hablan de emociones y no de afectividad; sin embargo, como mencionaremos más adelante, en nuestro caso nos referimos a afectos con el objeto de abarcar lo que generalmente se distingue como estado de ánimo, humor, emoción, etcétera.
} 
el humor y los estados de ánimos. ${ }^{2}$ La intencionalidad, desde nuestra caracterización, y siguiendo a De Sousa, no se refiere a un objeto o situación particular, sino a una cualidad que define el estado afectivo en cuestión, por lo que si nos referimos, por ejemplo, a lo que tradicionalmente se considera un estado anímico como la depresión, la intencionalidad, en este caso y en relación al escenario en cuestión, sería la cualidad depresiva.

Asimismo, la analogía entre estados afectivos y percepción nos permite comprender los casos en los que ante una misma situación el objeto formal afectivo para un agente, que experimenta un escenario determinado, puede ser terrorífico, y para otro, el objeto formal de su escenario puede ser excitante. En este sentido, el contexto cultural y la historia de vida del agente son ámbitos esenciales en la conformación de un escenario afectivo; ámbitos donde quizás deberíamos buscar dicha diferencia.

De lo expuesto, un punto central para aclarar es que los afectos no son meras proyecciones, sino que envuelven un momento determinado de la realidad, presentan ciertas facetas del entorno. Estos no están en la mente, no pertenecen a un espacio privado accesible únicamente al agente; como veremos más adelante, los estados afectivos involucran estados actitudinales, lo que supone ciertas formas de engranarse y comprometerse con el entorno. En este sentido, siguiendo a Solomon, el enojo, el estar enamorado o el desenamorarse, no es simplemente "ver" el mundo de manera diferente, es estar profundamente comprometidos (como opuesto a lo que simplemente nos sucede) con una situación. Producimos demandas y expectativas de gran alcance y proyectamos en la situación, en el entorno y en la persona, los posibles cumplimientos o frustraciones de esas demandas y expectativas. (2004, p. 83).

Así, al igual que la experiencia perceptual, entendida en un sentido no meramente fisiológico como simple estímulo y suscitación sino

\footnotetext{
${ }^{2}$ Generalmente se asume que las emociones se distinguen del humor y los estados de ánimo por su intencionalidad, es decir, por dirigirse a un objeto o situación particular; ésta sería la diferencia, por ejemplo, del miedo, que, según esta concepción, es siempre miedo de, y de la depresión, la cual, según la misma clasificación, carecería de objeto, por lo que no se puede considerar una emoción, sino un estado de ánimo. Asimismo, la distinción entre humor y estado de ánimo se basa en la temporalidad: el humor es un estado más pasajero que los estados de ánimos.
} 
como intelección del propio estímulo, los afectos también están engranados con el mundo, esto significa que el agente está en un entorno significativo y no simplemente en un entorno material. Asimismo, este entorno o realidad no solamente produce ciertos estados en el agente, sino que forma parte fundamental de su afectividad; hay una dependencia entre la naturaleza de la experiencia y la naturaleza de lo que es percibido; la experiencia, en cierta medida, traza la naturaleza de lo que es experimentado (Jackson, 1996, p. 205).

Entendidos de esta manera, los afectos, como análogos a la percepción y caracterizados a partir de la cualidad que los define, resaltan aspectos de la realidad que de otra manera permanecerían ocultos, por ejemplo, la cualidad de terrorífico, establecerá patrones de notabilidad en una situación determinada, destacando aspectos -tanto objetos, regularidades o relaciones de relevancia o irrelevancia entre éstos- que de otra forma pasarían desapercibidos.

\section{Afectividad y cognición. Vínculo entre afectividad y creencias}

Una consecuencia de lo dicho hasta aquí es que lo que consideramos un afecto en particular puede involucrar una cantidad de estados tan complejos, que tornan difícil un análisis unívoco. Como mencionamos, nuestra caracterización de estados afectivos involucra componentes culturales, psicológicos y fisiológicos, sin los cuales la conformación de "escenarios afectivos" se tornaría imposible. De aquí, que distintas áreas, como la psicología, la biología, la sociología, las neurociencias y la filosofía, entre otras, explicarán aspectos particulares de la afectividad; no obstante, debido a que los afectos no poseen un núcleo esencial, ninguna de ellas se torna más fundamental que la otra.

De acuerdo con esto, podemos decir, siguiendo a Hacking (1999), que los afectos son clases interactivas: "interactúan con las personas y sus comportamientos” (p. 215), dentro de contextos histórico-culturales específicos. En dicha interacción hay un mutuo fortalecimiento o una recíproca transformación, los afectos, como clases interactivas, refuerzan o modifican los estados actitudinales de los agentes, y a su vez, los agentes pueden fortalecer o modificar dichas clases acorde a las transformaciones en la cosmovisión de sus culturas.

Esto explicaría que muchos estados afectivos, adecuados en un determinado momento histórico-cultural, se tornen inadecuados en otro 
momento. Aquí radica, asimismo, el problema de muchas teorías sobre la afectividad fundamentadas en una concepción exclusivamente evolucionista, comprometidas con la tesis de que los afectos, al menos los considerados básicos o universales , ${ }^{3}$ tienen una historia basada en la adaptación y selección natural, sin embargo, como venimos afirmando, los afectos poseen elementos culturales que no pueden ser reducidos a aspectos biológicos, lo que torna necesario incorporar el contexto cultural en la explicación de su desarrollo y conformación.

Con esto, y siguiendo a Jones (2006), si los afectos fueran mecanismos de estímulo-respuesta, la sola idea evolucionista sería suficiente; sin embargo, como veremos a continuación, los afectos son mecanismo que interactúan con, y regulan otros mecanismo o ámbitos, que a su vez, están sujetos a otras interacciones y constreñimientos (p. 17).

Conforme a lo anterior, los afectos constituyen un ámbito compuesto, de manera fundamental, por el contexto cultural en el que se desarrolla. En dicha constitución, y en la propia manifestación de los procesos afectivos, pueden participar otros ámbitos de la cognición, como las creencias. Efectivamente, en la mayoría de los casos hay una relación íntima entre afectos y creencias, la cual, aunque no conduce a una concepción reduccionista de la afectividad, sí manifiesta la necesidad de tomar en cuenta otros estados cognitivos para una mejor comprensión de los procesos afectivos. En el caso del vínculo entre creencia y afectos, según Fridja y Mesquita (2000), se puede producir, por un lado, porque creencias previamente elaboradas provocan el surgimiento de ciertos afectos en el agente o, por el otro, porque los afectos estimulan y conforman ciertas creencias (p. 52). Así, procesos cognitivos como creencias, juicios o valoraciones, no sólo pueden ser un input o estímulo para el surgimiento de ciertos afectos, sino que muchas veces son el resultado de un proceso afectivo.

Para una mayor claridad, es oportuno detenernos en la noción de creencia que supone nuestro desarrollo. Desde nuestro tratamiento, y de acuerdo a la propuesta de Villoro (1982), la cualidad fundamental de las creencias es que son estados disposicionales, es decir, creer es,

${ }^{3}$ Los afectos básicos o universales se vinculan con la distinción entre afectos primarios y secundarios. Los básicos o universales corresponderían a los primarios, los cuales se caracterizan, en general, por ser estados innatos, cercanos a los actos reflejos, cuya explicación se basa, principalmente, en los aspectos fisiológicos del agente. Un ejemplo de afectos primarios es el miedo. Cfr. Damasio (1994). 
principalmente, "una disposición que se manifiesta en nuestro estar por entero en el mundo y no sólo en nuestra conciencia” (p. 34).

Con esto, se fija una distinción elemental entre las creencias y otros estados como las representaciones; acorde con la propuesta de Villoro, éstas son meras aprehensiones, descripciones o comprensiones del mundo (p. 62) pero carecen del elemento que compromete a un agente con lo representado; por el contrario, las creencias incluyen representaciones pero incorporan este aspecto disposicional, por el cual una creencia orienta la manera en que un agente se relaciona con el mundo.

En términos de Fridja y Mesquita, las creencias son estados por los que una persona está propensa a actuar de una determinada manera (p. 46); de aquí que tener una creencia $p$ es actuar y relacionarse con el mundo como si $p$ fuera verdadera. Así, poseer una representación de $p$ no es suficiente para orientarse en el mundo a partir de ella misma, se requiere el aspecto disposicional para que el agente tenga dicha propensión. Con esto, Villoro afirma:

Las creencias operan como una guía de mis acciones que me pone en situación, me "dispone" a responder de determinadas maneras y no de otras, en las más diversas circunstancias. Porque al creer en un hecho considero que ese hecho forma parte del mundo real y, por lo tanto, me relaciono con el mundo contando con su existencia (p. 33).

Este aspecto disposicional torna a las creencias estados sensibles a los afectos debido al carácter actitudinal de los procesos afectivos. Efectivamente, en términos de Fridja, Manstead y Ben (2000):

Hay una diferencia entre conocer y creer de significancia psicológica, esta es la manera en que ellas preparan al agente para actuar. Tener una creencia no es tanto la afirmación de tener un conocimiento verdadero como tomar el riesgo y estar preparado para la acción. Esto debería significar que las creencias son más sensibles a las emociones que al conocimiento ( $\mathrm{p}$. $4)$.

Con esto, sostenemos que hay una relación de ida y vuelta entre los afectos y otros estados cognitivos de un agente, como por ejemplo su estado doxástico. Esta relación bidireccional se manifiesta de forma 
evidente en ciertos casos de cambio o mutación afectiva; al respecto, siguiendo a Elster (1999), en muchas ocasiones hay una transformación en el estado afectivo del agente, el cual sería inexplicable sin la mediación de otro estado congitivo. Para mostrar esto, es iluminadora la alusión que hace Elster respecto a la manera en que se desenvuelve el proceso afectivo en uno de los personajes de G. Eliot en Middlemarch:

Porque Mr. Casaubon estaba celoso de Will Ladislaw, él deseaba comprometer a su esposa Dorothea para que no pudiera casarse con Will cuando muriese. Sin embargo, escribe George Eliot, 'Mr. Casaubon, sabemos, posee un sentido de rectitud y un honorable orgullo en satisfacer los requisitos de honor, lo cual lo forzó a encontrar otras razones para su conducta que la de los celos y el rencor' (G. Eliot Middlemarch, cap. LxiI, en Elster, 1999, p. 350).

En este proceso, afirma Elster, hay un cambio afectivo mediado por creencias, intenciones y valoraciones, lo que provoca una "metaemoción", o en nuestros términos, un "meta-afecto", en este caso, la vergüenza de sus celos. (p. 350).

No obstante, esto deja pendiente el tema de lo que se conoce como persistencia afectiva, es decir, aquellos casos en los que ante la adquisición o cambio de una creencia, que debería conducir a la transformación de un cierto estado afectivo, éste permanece inalterable. La tesis habitual a la que encaminan estos casos es que hay una contradicción entre la creencia que afirma poseer un agente y el estado afectivo que manifiesta en relación a la situación que involucra dicha creencia, por ejemplo, un agente puede afirmar, basado en las estadísticas sobre accidentes relacionados con distintos medios de transporte, que el transporte más seguro para viajar es el aéreo, y sin embargo, el agente en cuestión teme volar. Según algunos desarrollos, estos casos muestran que la idea de que existe una interacción entre los afectos y otros estados cognitivos es errónea.

Contrario a este tratamiento, nuestra interpretación indica que el problema surge por una equívoca manera de enfocar el problema, la cual se orienta a examinar el estado afectivo ante la creencia del agente en vez de enfocarse en la propia creencia que el agente afirma poseer. Esta aseveración está íntimamente vinculada con nuestra noción de creencia cuyo rasgo fundamental, como expusimos, es su componente 
disposicional. Dicho componente no significa mera disposición hacia una proposición, sino una disposición hacia el mundo, lo que permite afirmar que las creencias se desempeñan como orientadoras o guías en la interacción del agente con su entorno.

Así, en el ejemplo sobre la creencia de un agente respecto al transporte aéreo, se presenta una contradicción entre la creencia que asume tener el agente, "los aviones son seguros", y la disposición hacia el transporte aéreo; disposición que manifiesta una relación con el entorno, contraria a la que se establecería en caso de poseer la creencia "los aviones son seguros". Esta incongruencia puede encontrar diversas explicaciones, pero todas tendrán su fuente en la propia creencia o red de creencias del agente. Así, en nuestro ejemplo, la incongruencia puede deberse al autoengaño o a que, dentro de la red de creencias que posee el agente, su estado disposicional es orientado por creencias con mayor peso o relevancia que la creencia sobre la seguridad del transporte aéreo, como por ejemplo la creencia de que aunque las posibilidades de un accidente aéreo son muy bajas en relación a otros medios de transporte, esa posibilidad mínima puede presentarse, y en caso de ser así, las probabilidades de sobrevivir son prácticamente nulas.

Con esto, lo que queremos acentuar es que el problema debe tratarse como una aparente contradicción en la estructura misma de la creencia o red de creencias, entendida como un estado disposicional, y no como una contradicción entre distintos estados del agente, como por ejemplo, su estado de creencias y su estado afectivo. Ciertamente, como advertimos, tener una creencia es tener lo que se afirma por verdadero, en palabras de Villoro:

[... ] estar dispuesto a que mi relación con el mundo esté determinada por ese objeto o situación [...] una proposición comprendida pero no aseverada, no suscita expectativas acerca de mi comportamiento posible, porque no informa nada acerca de lo que pudiera encontrarme en el mundo real, no determina para nada mis propensiones a actuar en ningún sentido [... ] (p. 62).

En el lenguaje cotidiano, creer y sentir se utilizan como sinónimos, no sólo creo que $p$ sino que siento que $p$; en este sentido la creencia ha de vivirse más que pensarse. Todo esto nos conduce a que las creencias 
no radican en el ámbito mental de un agente, como pretende el idealismo, sino que radica en este aspecto disposicional, lo que la convierte en un estado primordialmente público.

Según Jones, la agencia, y la toma de decisiones que acompaña al concepto de agencia, requiere de procesos informacionalmente abiertos, es decir, requiere integrar información proveniente de una variedad de ámbitos cognitivos (2006, p. 18). Los ambientes complejos de los agentes requieren la participación de distintas funciones cognitivas que le permitan acomodarse a las variaciones del contexto; en este sentido, los afectos son estados abiertos a otros ámbitos cognitivos como las creencias, los juicios y los valores.

\section{El agente epistémico afectivo}

A partir de lo dicho hasta aquí, un problema que suele plantearse es cómo distinguir entre experiencias afectivas y no afectivas, o en términos de lo que hemos expuesto, cómo distinguir entre escenarios paradigmáticos afectivos y escenarios no-afectivos. En este sentido, nuestra visión adopta una postura más radical al asumir que no existe experiencia sin afectividad, es decir, que los estados afectivos están presentes en toda experiencia como condición de posibilidad para que dicha experiencia sea posible.

Esta manera de entender la experiencia y su vínculo con la afectividad se encuentra presente en el concepto de "cualidad estética" propuesto por Dewey (1934). Con éste, Dewey alude primordialmente al ámbito de la afectividad, la cual, en palabras del autor, "lanza tentáculos para lo que es afín, para las cosas que la alimentan y la conducen a su cumplimiento" (p. 78). ${ }^{4}$ Acorde con esto, las cualidades estéticas fungen como elemento organizador y otorgador de sentido al proceso experiencial.

Siguiendo esta línea de pensamiento, nuestra propuesta es que la experiencia está afectivamente engranada con el mundo; no obstan-

\footnotetext{
${ }^{4}$ En esta cita el autor se refiere directamente a "emoción" y su papel en la producción y vivencia de la obra artística. Como veremos a continuación, esta función que le otorga a los estados afectivos es muy cercana a la que le otorga a la cualidad estética, es por esto que asumimos una cierta analogía entre ambos conceptos. No obstante, la diferencia parece radicar en que la cualidad, como elemento temporalmente anterior en el desarrollo de la experiencia, individualiza a la emoción que predominará en el proceso experiencial, es decir, que la cualidad dominante define el significado de los sentimientos o emociones experimentados.
} 
te, este engranaje afectivo no requiere ser reflexivo. Efectivamente, podemos tener ciertos afectos sin ser, en el momento, reflexivamente conscientes de éstos. Esta imagen conserva cierta semejanza con la distinción propuesta por Hume (1739) entre pasiones calmas y pasiones violentas, donde las primeras son apenas perceptibles como tales y serán conocidas más por sus efectos que por su sentimiento inmediato o sensación (p. 14, vol.II). Como afirma Hume, muchos han supuesto que la razón por sí misma puede motivar debido, en parte, a que algunas pasiones son calmas; desde que estas pasiones no causan desorden en el alma, las motivaciones que ellas provocan son tomadas por determinaciones de la sola razón (p. 190, vol. II). Sin embargo "La razón por sí sola jamás puede ser un motivo de una acción de la voluntad [...] la razón jamás puede oponerse a la pasión en la dirección de la voluntad" (p. 185, Vol. II).

Basados en este pensamiento, suponemos que no hay experiencia, y por lo tanto, dada nuestra caracterización de experiencia, tampoco hay agencia, sin afectividad. Desde nuestra visión, estas pasiones a las que alude Hume o, en nuestros términos, los afectos irreflexivos no sólo son fuente de motivación sino que constituyen escenarios paradigmáticos estableciendo patrones de notabilidad y posibilitando los procesos cognitivos en general.

Estas ideas se sostienen a partir de una concepción particular de agencia epistémica asentada en el supuesto de que todo conocimiento es resultado de un proceso agencial alcanzado bajo ciertas condiciones y no un estado que simplemente "se tiene". Con esto, queremos señalar, en coherencia con Hookway (2006), que:

El razonamiento es un tipo de actividad dirigida a ciertos fines o metas $[\ldots]$ desde que el razonamiento es una actividad dirigida a metas, las normas que gobiernan el razonamiento y la investigación incluirán normas de razón práctica: estamos evaluando estrategias para resolver problemas y la efectividad del agente para llevar a cabo sus estrategias (pp. 99-100).

De aquí, acorde con la línea pragmatista que orienta este trabajo, la noción de agencia está íntimamente vinculada con una situación problemática y con el deseo de restablecer un orden. Este supuesto torna central, para entender la idea de agencia, la noción de comprensión 
entendida como un logro epistémico fundamental junto a la noción de conocimiento.

Por comprensión, siguiendo a Broncano (2006a), no aludimos a un proceso principalmente intelectual, sino a una forma de ser en el mundo; con esto, el concepto de comprensión cuestiona la centralidad del conocimiento, aunque no niega su relevancia, en las prácticas epistémicas, en el sentido de que ahora se torna también fundamental la manera en que un agente se apropia y da sentido a su entorno.

Son varios los autores que asumen la conveniencia de incorporar la noción de comprensión en el marco epistemológico; esto se debe, en cierta medida, a que, como afirma Elgin (1996), la comprensión es un término más inclusivo que la idea de conocimiento:

[... entendemos reglas y razones, acciones y pasiones, objetivos y obstáculos, técnicas y herramientas, formas, funciones, como también hechos. También comprendemos cuadros, palabras, ecuaciones y patrones. Por lo general, éstos no son logros aislados, sino que se funden dentro de la comprensión de un tema, una disciplina o de un campo de estudio (p. 123).

La comprensión, señala Elgin, involucra una cierta relevancia epistémica que el conocimiento o la creencia verdadera parecen dejar a un lado. Así entendida, la comprensión requiere de una cierta sensibilidad agencial, la cual, siguiendo el desarrollo de Broncano, involucra la participación afectiva. En términos de Greco (1999), dicha sensibilidad alude a una capacidad del agente para saber que cierta evidencia constituye una indicación confiable de que determinada creencia es verdadera; es decir, la sensibilidad para "ver" y aceptar cierta evidencia como relevante (p. 289).

La sensibilidad incluye ciertos hábitos y disposiciones afectivas constitutivas de los rasgos de carácter del agente. Esto posibilita entender la sensibilidad como la propone Broncano, esto es, la sensibilidad respecto a los requerimientos de una situación es un rasgo vinculado con la capacidad, propia de todo agente, de dar sentido:

La habilidad para dar sentido no es independiente de ser un agente. Por el contrario, uno se convierte en agente como resultado de ser inmerso en la realidad a través de una cierta forma de sentido y es motivado por la forma de entender (Broncano, en prensa, p. 8). 
La centralidad de la comprensión y de la sensibilidad como constitutivos de la agencia tornan a los afectos un ámbito fundamental en los procesos epistémicos. Como vimos, los afectos se asemejan a la percepción en el sentido de que constituyen "maneras de ver", desde esta caracterización, los afectos participan en el ámbito epistémico al establecer patrones de relevancia o notoriedad influenciando en los procesos inferenciales y en la conformación de creencias; es decir, siguiendo a Hookway (2006), hay una cierta inmediatez afectiva en los procesos cognitivos.

Una consecuencia esencial respecto a esta inmediatez afectiva es que, si bien los afectos participan en procesos cognitivos, como por ejemplo en los procesos inferenciales, no figuran en la justificación argumentativa. Es decir, dicha participación afectiva revela, a fin de cuentas, que dichos procesos no son del todo explícitos. No obstante, a pesar de no figurar en la justificación, los estados afectivos son necesarios para la cognición constituyendo el trasfondo que posibilita dichos procesos, al punto, como afirma Hookway, de que sin esta inmediatez afectiva sería imposible la adquisición de creencias. ${ }^{5}$

La agencia, según lo dicho hasta aquí y en palabras de Broncano, es esencialmente "una facultad fiable que adscribimos a seres que son capaces de modificar su entorno de acuerdo con sus necesidades y deseos" (2006a, p. 11), esta facultad incluye la capacidad de aprendizaje como un elemento primordial del proceso de transformación intencional del entorno. Esta caracterización alude a dos aspectos esenciales. El primer aspecto, que refiere a las facultades agenciales, involucra las denominadas corrientes confiabilistas y responsabilistas sobre la epistemología de la virtud; el segundo aspecto, manifiesta la importancia de un cierto tipo de conocimiento, conocimiento agencial, que resulta del proceso agencial y que, a su vez, es requerido para que dicho proceso sea posible.

Respecto al primer aspecto, la propuesta confiabilista sobre las facultades agenciales refiere, en palabras de Broncano, "a las condiciones fácticas de actuación, las cuales incluyen ciertas condiciones neuromotoras $[\ldots]$ la fiabilidad tiene que estar sostenida por ciertos rasgos

${ }^{5}$ Cfr. Hookway 2003. 
de diseño de los mecanismos que están involucrados en la acción" (p. 11), estas facultades, que incluyen no sólo a las neuromotoras, sino que implican a su vez hábitos, actitudes, habilidades y afectos, son condiciones necesarias para que el vínculo entre intención, medios y resultados sea exitoso. En cuanto al responsabilismo, alude a la naturaleza activa del agente, reconociendo los procesos creativos y de elección involucrados en la actividad de conocimiento.

El punto central es que dichas facultades son un ámbito nuclear que debe tenerse en cuenta para la valoración epistémica. En relación a esta idea, Broncano afirma:

Desde el punto de vista del conocimiento implicado en el control de la acción, se exige que el agente conozca algo acerca del control de su conducta. En relación con este requisito, una consecuencia es que el agente tiene que cumplir una determinada condición epistemológica para lograr efectivamente el control de manera eficiente y no por suerte o don de la naturaleza o del entorno (p. 11).

Como señalamos, dicho conocimiento es posibilitado por la propia experiencia entendida como investigación, transformación y aprendizaje; en términos de Dewey:

Tan pronto como un niño comienza a esperar, empieza a usar algo que está ocurriendo ahora como un signo de algo que va a seguir; está juzgando aún cuando sea de una manera muy simple. Pues toma una cosa como evidencia de otra y así reconoce una relación. Todo futuro desarrollo, por complicado que pueda ser, es sólo una extensión y un refinamiento de este simple acto de inferencia (1916, p. 129).

Así, acorde a lo desarrollado hasta aquí, lo afectivo, lo cognitivo y los actitudinal son elementos constitutivos de un mismo proceso, o en términos del mismo Dewey, todo afecto se constituye de tres partes: la fisiológica, la cognitiva y la actitudinal; con esto, la afectividad es una forma de conducta, una tendencia a comportarse de determinada manera (1984, p. 180).

Desde estos supuestos, afirmamos que el componente afectivo está al centro de toda experiencia, como condición de posibilidad, incluida la experiencia epistémica. Esta idea nos obliga a criticar las distinciones 
absolutas a priori entre medios y fines, pasado, presente y futuro, valores y hechos, subjetivo y objetivo; desde estas críticas, los medios no son un ámbito neutral en relación a los fines; por el contrario, medios y fines se constituyen mutuamente dentro del proceso agencial. En este proceso, el establecimiento de fines sólo puede realizarse en términos de las condiciones mediante las cuales adquieren existencia, es decir, a partir de la consideración de medios; "en los deseos e intereses que determinan ciertos fines entran necesariamente proposiciones en las que se evalúan cosas (actos y materiales) como medios" (Dewey 19381939, p. 88); es decir, es un proceso anclado en la transacción entre un agente y el entorno.

En el proceso agencial, que va constituyendo los medios y los fines, el pasado y el futuro se hacen presentes como ámbitos posibilitadores de la experiencia del agente. En términos de Broncano, el pasado, como contraposición de lo que podría haber sido, se hace presente a través de la nostalgia, el resentimiento, el duelo; en este sentido, afirma Broncano, todo juicio sobre el pasado es contrafáctico, es "el juicio de lo que fue, en tanto que necesario, contra el trasfondo de lo que podría haber sido"(2006b, p. 33). Respecto al futuro, lugar donde confluyen los proyectos de vida, existe como una posibilidad sobre la que opera la voluntad; estas posibilidades, continúa Broncano, actúan sobre el presente como deseos, temores, intenciones, compromisos, etcétera (p. 20).

El agente epistémico, según esta idea, y acorde con la propuesta de Dewey, involucra actos reflexivos basados en experiencias pasadas, las cuales, a su vez, constituyen las posibles maneras de ajuste o "transacción” entre mente-mundo, es decir, forman parte de la propia proyección; en palabras de Dewey, las experiencias pasadas intervienen en la consideración "del efecto de lo que ocurre sobre lo que puede ser, pero que no es todavía” (1916, p. 130). En esta idea está el supuesto de fondo de que ciertos procesos inferenciales no son ajenos a la experiencia de un agente sino que, por el contrario, se constituyen dentro de ella.

Esta interpretación nos conduce a una manera particular de entender la experiencia con fines epistémicos y la racionalidad implicada en dicha experiencia. Desde este desarrollo, la experiencia epistémica consiste, fundamentalmente, en investigación basada en metas confor- 
madas a partir de un problema específico. Entendida así, y conforme a Hookway:

[...] el objetivo de evaluación epistémica descansa en nuestra habilidad para llevar a cabo la investigación, razonar efectivamente y resolver problemas $[\ldots]$ se evalúa la competencia epistémica de un agente bajo el reconocimiento de que el razonamiento es una actividad con metas y propósitos basada en un sistema de hábitos, capacidades, actitudes, habilidades, entendido como proceso "subdoxástico" que posibilitan las condiciones para la reflexión y razonamiento (2006, p. 103).

A partir de este entendimiento, Hookway propone redefinir el ámbito de valoración epistémica; en esta redefinición, la justificación deja de ser el foco central de la epistemología, siendo ahora su objetivo fundamental la investigación, es decir, las actividades para resolver problemas. Para esta epistemología la preocupación fundamental es cómo las estrategias y los métodos nos conducen a alcanzar nuestras metas intelectuales.

Esta propuesta apunta a una epistemología centrada en el agente y en los procesos de adquisición de conocimiento; a una epistemología, en palabras de Broncano y Vega (2011), que no sólo evalúe el éxito alcanzado (entendido éste quizás como conocimiento, certeza, etc.) sino los medios por los que un agente alcanza dicho éxito, es decir, un enfoque epistemológico que considere la manera en que "un agente se compromete con la tarea de conocimiento" (p. 2).

Una corriente epistemológica que responde a estos cambios es la "epistemología de la virtud"; a grandes rasgos este enfoque epistemológico parte de un cambio de foco centrándose en las características o rasgos intelectuales de los agentes. En palabras de Sherman y White (2003) "parte del atractivo de esta propuesta epistemológica es este cambio de enfoque desde la justificación de creencias individuales al estatus global del conocedor como una persona intelectualmente virtuosa" (p. 34).

Una manera de comprender este cambio de enfoque de la epistemología de la virtud es a partir de una comparación con el análisis de la epistemología tradicional; una línea epistemológica tradicional define una creencia epistémicamente justificada como aquella que es soportada por cierta evidencia, donde dicha evidencia es completamente inde- 
pendiente de las propiedades del agente que posee la creencia; para esta postura la virtud referirá a la disposición del agente para creer acorde con una evidencia definida independientemente del agente, es decir, definida sin tomar en cuenta las virtudes del agente. Por el contrario, una epistemología enfocada en las virtudes intelectuales cambia el orden de análisis, en donde la justificación de una creencia es aquella que manifiesta las virtudes intelectuales del agente.

La noción central dentro de este enfoque epistemológico es la de virtud, concepto que tiene una larga trayectoria dentro del ámbito de la ética, pero que en el campo de la epistemología ha recibido escasa atención. Si bien no nos adentraremos en este tema, creemos importante señalar, en relación a nuestra propuesta, que desde que asumimos que el razonamiento es un tipo de actividad dirigida a determinados fines y que lo esencial del acto de investigación es la resolución de problemas, estamos admitiendo una especie de paralelismo entre ciertos desarrollos en el campo de la ética y en el ámbito de la epistemología, en particular, una cierta semejanza entre la ética y la epistemología de la virtud.

Para comprender mejor estas ideas, Hookway, después de proponer un ejemplo en donde el agente se pregunta si 71 es un número primo, menciona algunos aspectos distinguibles en el proceso cognitivo que desarrolla el agente:

1) ¿Cuál es la meta de razonamiento?, ¿qué problema está tratando de resolver?; 2) ¿qué estrategia emplea para resolver el problema? ¿qué tan efectiva es esta estrategia?; 3) ¿cómo planea llevarla a cabo? ¿̇es ésta una manera efectiva de ejecutarla? ¿se realiza en la manera en que se había planeado?; 4) ¿qué capacidades (intelectuales) intervinieron?

En el ejemplo propuesto, las respuestas serían:

1) La meta es establecer si 71 es un número primo; 2) la estrategia es dividir sucesivamente el 71 entre los números primos menores o iguales a su raíz cuadrada. Esta es una estrategia efectiva; 3) Los medios elegidos para llevar a cabo esta estrategia es ver si 71 es divisible por algún número primo menor a 10. Esta es una buena manera de llevar a cabo la estrategia y es exitosamente ejecutada; 4) En la solución de este problema, el agente confía en sus habilidades para hacer el cálculo mental, para identificar números primos pequeños, para hacer un seguimiento de los resultados de los cálculos anteriores, etc. Estas habilidades pueden incluir el conocimiento sistemático detallado de (por ejemplo), la aritmética, pero también es 
probable que involucre a la heurística confiable, hábitos fiables de cómputo, etc. (2006, p. 99).

Como podemos apreciar, el análisis toma en cuenta el proceso agencial en su totalidad, la evidencia que soporta o justifica la creencia, en este caso que 71 es un número primo, manifiesta los rasgos intelectuales del agente, es decir, se enfoca en el estatus integral del agente epistémicamente virtuoso. Esto incluye, de manera esencial, las metas y los medios que se establecen a partir de un problema que orienta la investigación; con esto, se torna necesario incorporar normas de la razón práctica en la normatividad epistémica.

Un aspectos esencial, es que este enfoque permite reconocer el papel de los afectos en ámbitos epistemológicamente relevantes, como por ejemplo en la evaluación epistémica. Con esto, queremos destacar que el papel de los afectos en la cognición no se limita a una función únicamente heurística. Nuestro supuesto, acorde con lo visto en los apartados anteriores, en particular con la caracterización de los afectos como "maneras de ver" o "patrones de notabilidad", es que la afectividad forma parte de este carácter intelectual, o dicho de otra manera, hay un aspecto afectivo en la conformación de la virtud que posibilita que ciertas habilidades y capacidades del agente se ejerzan de manera efectiva para la investigación y el logro de fines de forma exitosa.

Lo anterior cobra mayor relevancia si consideramos que el agente al cual nos referimos es encarnado en permanente interacción con dinámicos, impredecibles y limitados recursos, y en general, inserto en ambientes sociales donde debe satisfacer una determinada clase de posibles metas. En este medio, los afectos, o al menos una clase de ellos, son un mecanismo propio de los agentes para tratar mejor con dichos ambientes. Al respecto, Cañamero (2002) establece una distinción entre agentes con un bajo nivel de autonomía y aquellos con un alto nivel en relación a la complejidad del comportamiento vinculado a las características del entorno. Así, en un nivel bajo, el agente es autónomo ya que puede actuar por sí mismo sin necesidad de comando por parte de otro agente; sin embargo, esta autonomía puede consistir en compor- 
tamientos muy simples, tales como la respuesta reflejo a un estímulo externo. ${ }^{6}$

En los casos de un alto nivel de autonomía, el agente puede elegir si poner o no atención y reaccionar a un estímulo del ambiente en función de la relevancia para su comportamiento y sus metas. En este nivel, el problema fundamental es el de la selección. Por ejemplo, en Inteligencia Artificial se ha logrado incorporar ciertas funciones a los agentes artificiales; no obstante, estos modelos sólo pueden trabajar de manera satisfactoria en ambientes moderadamente dinámicos, pero cuando incrementa el dinamismo del entorno su capacidad para trabajar es deficiente. Dichos ambientes más complejos y cambiantes requieren una veloz adaptación ante los rápidos cambios de las circunstancias del entorno; es decir, se requiere agentes flexibles con la capacidad de modificar sus metas y sus comportamientos (Cañamero 2002, pp.115-116).

Con esto, y siguiendo a Damasio, asumimos que el poder de la razón y la capacidad afectiva se deterioran juntos. Los sistemas relacionados con los afectos, la atención y la memoria funcional interactúan de manera íntima constituyendo una fuente esencial que anima el pensamiento y el razonamiento. El mundo sin afectos sería un mundo descolorido sin ningún elemento que impulse a la decisión y estimule el razonamiento. En palabras de Johnson-laird (1993), "para decidir, hay que juzgar; para juzgar, hay que razonar; para razonar, hay que decidir sobre qué se razona" (p. 109). Los afectos orientan respecto al qué razonar, acotando el abundante caudal de información y opciones que se le presenta al agente, dicha función incluye, por supuesto, la decisión de la estrategia lógica a seguir para producir inferencias válidas.

\section{Referencias}

Aristóteles, 1993, Ética Nicomáquea. Ética Eudemia, Gredos, Madrid.

Broncano, F., 2006a, "Consideraciones epistemológicas acerca del 'sentido de agencia”, en Logos. Anales del Seminario de Metafísica, vol. 39, pp. 7-27.

${ }^{6}$ Cañamero 2002, pp. 115-116. Según nuestra caracterización, éstos no serían agentes, ya que la definición aquí planteada, a diferencia de la de Cañamero, no se basa en los efectos casuales de ciertos actos sino en la sensibilidad agencial que posibilita ciertos logros agenciales y cognitivos como la comprensión, lo que involucra un cierto éxito en la interacción con el medio. No obstante, lo que pretendemos mostrar con la cita es la relevancia de los afectos para que dicha interacción sea exitosamente posible en ambientes complejos. De aquí que no nos centremos en este distanciamiento con la autora. 
- , (En prensa) "Agency and the sense of an ending".

Cañamero, L. D., 2002, "Designing Emotions for Aactivity Selection in Autonompus Agents" en Trappl, Petta y Payr (ed.) 2002.

Calhoun, C. y R. C. Solomon, (comp.) (1984) ¿Qué es una emoción? Lecturas clásicas de psicología filosófica, FCE, México.

Damasio, A., 1994, Descarte's error. Emotion. Reason and the human brain, G.P. Putnam's Sons, Nueva York.

Dewey, J., 2004, Democracia y educación, Ediciones Morata, Madrid.

—, 2008, Teoría de la valoración, Ediciones Siruela, Madrid.

—, 2000, La miseria de la epistemología. Ensayos de pragmatismo, Biblioteca nueva, Madrid.

— , 1996, "La teoría de la emoción" en Calhoun y Solomon 1996.

De Paul, M. y L. Zagzebski (ed.), Intellectual Virtue. Perspectives from Ethics and Epistemology, Oxford University Press, Oxford.

De Sousa, R., 1987, The rationality of emotions, MIT Press, Massachusetts.

—, 2004, "Emotions: What I Know, What I'd Like to Think I Know, and What I'd Like to Think" en Solomon 2004.

Duhem, P., 1953 "Physical Theory and Experiment", en Feigl y Brodbeck 1953.

Elster, J., 1999, Alchmies of the mind: Rationality and the emotions, Cambridge University Press, Cambridge.

Faucher, L y Ch. Tappolet, 2006, The modularity of emotions, Canadian Journal of Philosophy, Supplementary vol. 32; University of Calgary Press, Canadá.

Feigl, H. y M. Brodbeck, 1953, Readings in the philosophy of science, University of Minnesota, Minnesota.

Fodor, J. A., 1983, The modularity of mind, MIT Press, Massachusetts.

Fridja, N. H. y B. Mesquita, 2000 "Belief through emotions" en Fridja, Manstead y Bem (ed) 2000.

Fridja, N, A. Manstead y S. Bem (ed), 2000, Emotions and Belief. How Feelings Influence Thoughts, Cambridge University Press, Cambridge.

Griffiths, P. E., 2004, "Is Emotion a Natural Kind?", en Solomon 2004.

Hacking, I., 2001, ¿̇La construcción social de qué?, Paidós, Barcelona.

Hookway, C., 2006, "Epistemology and Inquiry: the Primacy of Practice", en Hetherington 2006.

Hetherington, S. (ed.), 2006, Epistemology futures, Oxford University Press, Oxford.

Hume, D., 2001, Tratado de la naturaleza humana, tomo II, Ediciones Gernika, México.

Jackson, F., 1982, “Epiphenomenal Qualia”, en The Philosophical Quarterly, vol. 32 , no. 127 , pp. $127-136$.

— , 1996, "The Primary Quality View of Color Source", en Noûs, vol. 30, pp. 199-219. 
Johnson-Laird, P. N. y E. Shafir, 1993, "The interaction between reasoning and decision making: an introduction", en Cognition, vol. 49, pp. 1-9.

Jones, K., 2006, "Quick and Smart? Modularity and the Pro-emotion Consensus”, en Faucher y Tappolet 2006.

Nussbaum, M., 1996, "Aristotle on emotion and rational persuasion", en Rorty 1996.

Polanyi, M., 1958, Personal Knowledge, Routledge \& kegan Paul, EUA.

Prinz, J. J., 2006, "Is emotion a form of perception?", en Faucher y Tappolet 2006.

Pylyshyn Zenon, W. (ed.), 1987, The robots dilema: The frame problem in artificial intelligence, Library of Congress, EUA.

Rorty, A. O., 1996, Essays on Aristotle's Rhetoric, University of California Press, California.

Sherman, N. y H. White, 2003 "Intellectual Virtue: Emotions, Luck, and the Ancients", en De Paul y Zagzebski 2003.

Solomon, R. C., 2004, Thinking about feeling. Contemporary Philosophers on Emotions, Oxford University Press, Oxford.

—, 2007, True to our feelings: what our emotions are really telling us, Oxford University Press, Oxford.

Stroud, B., 2000, The quest for reality: subjectivism and the metaphysics of color, Oxford University Press, Oxford.

Trappl, R, P. Petta y S. Payr (ed.), 2002, Emotions in humans and artifacts, MIT Press, Massachusetts.

Villoro, L., 1982 Creer, saber, conocer, Siglo xxi, México.

Recibido el 6 de Junio de 2012

Aceptado el 23 Junio de 2012 